\title{
Extensive spectroscopic data for multiply ionized scandium: Sc III to Sc XxI ${ }^{\star}$
}

\author{
G. Massacrier ${ }^{1,2}$ and M.-C. Artru ${ }^{1,3}$ \\ 1 CNRS, UMR 5574, Centre de Recherche Astrophysique de Lyon, 69364 Lyon, France \\ 2 Université de Lyon, 69003 France; Université Lyon I, Observatoire de Lyon, 69230 Saint-Genis-Laval, France \\ e-mail: gmassacr@ens-lyon.fr \\ 3 Université de Lyon, 69003 France; Ecole Normale Supérieure de Lyon, 69007 Lyon, France \\ e-mail: artru@ens-lyon.fr
}

Received 24 July 2011 / Accepted 7 December 2011

ABSTRACT

Context. Spectroscopic data for scandium is sparse, while an extensive set is needed to introduce this element into stellar opacity calculations and, more importantly, into stellar models dealing with radiative diffusion.

Aims. Our goal is to provide extensive energy levels and radiative transitions data for nineteen ionization stages of scandium relevant to stellar interiors, namely from Sc III to Sc XXI.

Methods. We used the FAC code. This code provides ab initio theoretical values for energy levels in $j j$-coupling and oscillator strengths of all permitted transitions. Detailed correspondences are established with compiled data from the National Institute of Standards and Technology (NIST) database, to locate as much as possible the observed levels and lines at their experimental values and to estimate the quality of our data. Comparison were also made with the spectroscopic data delivered by Kurucz.

Results. The theoretical data retained in tables include 21842 levels and more than two millions transtions. By comparison, the NIST compilation gives a total of 820 levels and $677 g f$-values. The good agreement obtained when comparing the new data with those of the NIST compilation demonstrates their quality. This work on scandium shows that the FAC code is efficient in providing spectrocopic data that are unavailable from laboratory analyses but necessary for accurate simulations of stellar plasmas.

Key words. stars: abundances - stars: chemically peculiar - atomic data - opacity

\section{Introduction}

Atomic and spectroscopic data are an essential and basic ingredient of studies in a wide range of research fields including astrophysics. In particular, stellar structure and evolution studies depend critically on these data. Significant theoretical and experimental efforts continuously aim to provide data of high accuracy, although there is also a need to expand the extent of data, as much-needed data are often absent from databanks. Though much progress has been made in terms of computing power, a compromise is still necessary between high accuracy, which is time consuming even for a few levels or transitions of one given ion, and completeness.

This work is motivated by advances in the description of the radiative diffusion in stars, owing in particular to this increasing amount of available atomic data. The importance of atomic inputs in obtaining correct opacities is widely recognized, but stellar models including radiative diffusion, initially aimed at understanding chemically peculiar stars, depend even more on the quality and completeness of these atomic input data. The data currently used are taken from the Opacity Project (Seaton et al. 1992, 1994), or sometimes provided by OPAL (Rogers \& Iglesias 1992). In an attempt to quantify whether abundance anomalies in Am stars originate from just below the superficial hydrogen convection zone or from below a (possible) deeper iron one, LeBlanc \& Alecian (2008) highlighted the key role

* Full Tables 4-6 and extensive line lists for each ion are only available in electronic form at the CDS via anonymous ftp to cdsarc.u-strasbg.fr $(130.79 .128 .5)$ or via

http://cdsarc.u-strasbg.fr/viz-bin/qcat?]/A+A/538/A52 of scandium and calcium. However, as pointed out by these authors, the available atomic data for scandium are quite sparse, or even absent from atomic databanks. A far more complete set is required to assess the validity of their conclusions.

In this paper, we present the results of a comprehensive computation of energy levels and transition data for nineteen ions of scandium, namely Sc III to Sc XIX, utilizing the Flexible Atomic Code (Gu 2003, 2008). Several thousands of fine-structure levels and millions of transitions have been computed, many of which have been made available electronically in the form of tables. Much of this effort has actually been made to assess the quality of the delivered data. The data itself was validated by means of an exhaustive comparison of our theoretical results with all available experimental data from the National Institute for Standards and Technology (NIST), which attests to the accuracy of the present work. This also allows us to assign exact wavelength values to the observed lines.

This paper is organized as follows. Section 2 explains our strategy to construct the atomic structure of each ion and select configurations. The comparison with experimental data and discussion of the accuracy of our results is performed in Sect. 3. Section 4 describes the final selected data and the content and organization of the delivered tables, and our Conclusions are given in Sect. 5.

\section{Procedure for theoretical calculations}

Our aim in the present work is to build a representation of the atomic structure and spectrum of scandium that is as complete as possible in view of astrophysical applications. We intend to 
deliver data for all energy levels lying below the first ionization threshold of a given ion, except for the principal Rydberg series, which is computed up to the principal quantum number $n=10$. This is in contrast to usual atomic structure computations, which concentrate on a few configurations and their transitions. Even for a not-too-heavy element such as scandium, our procedure generates and processes thousands of levels and millions of transitions in a sensible and controlled way.

The Flexible Atomic Code (FAC) is a relativistic configuration interaction program based on $j j$-coupling that allows these large-scale computations with the inclusion of up to thousands of fine-structure energy levels for each ion ( $\mathrm{Gu} 2003$, 2008). Though quite recent, FAC has become of widespread use in various research fields, and its precision has been widely tested by experiments (for instance: Aggarwal et al. 2010; Aggarwal et al. 2011; Clementson et al. 2010; Hahn et al. 2010; Wei et al. 2010). The versatility and speed of FAC allowed us by running numerous test cases to systematically construct a coherent and homogeneous set of data for nineteen ions of scandium.

Our strategy was to reconstruct stage by stage recombined ions starting from H-like Sc xxi down to Sc III. Once the atomic structure of an ion is settled an electron is added to its low lying configurations so as to determine whether the levels of the recombined ion fall below or above the ionization limit. All configurations leading to some levels below the ionization limit are retained. Supplemented with some of the above-threshold configurations, they are used in a single run including configuration interactions. Several test minimizations are performed using the ground configuration, as well as some excited ones to obtain the losest match to experimental data (see below). Once the energy level structure is set, radiative data for bound-bound transitions are computed. Only electric dipole (E1) transitions are considered.

Table 1 describes the configuration set involved in our representation of each scandium ion. The configurations that are included in the computation of electronic states but lead to energy levels above the limit are not mentioned. We note that some electronic states are highly mixed with no clear prominent contribution from a single given configuration. In addition to its Rydberg series, an ion may require several dozens of configurations to be described completely. Some of them have several open subshells and may generate a broad range of fine structure levels: for instance, in Sc vi the configuration $3 \mathrm{~s} 3 \mathrm{p}^{3} 3 \mathrm{~d}^{2}$ consists of 202 levels with a total degeneracy of 1390; those levels are spread over more than $47 \mathrm{eV}$ of which $34 \mathrm{eV}$ lie below the ionization limit. These configurations are loosely connected to ground states through one electron jump transitions and are consequently absent from experimental databases. They do however matter because of the configuration interaction. Owing to their statistical weight, one may also anticipate some influence in the calculation of population equilibrium.

\section{Comparison with available experimental data}

To assess the quality of our computations, we performed systematic comparisons with the reference data obtained from the National Institute of Standards and Technology website ${ }^{1}$ (Ralchenko et al. 2010). The NIST atomic spectra database provides a compilation of (mainly) experimental levels and wavelengths for all scandium ions. Reference papers describing this compilation are Sugar \& Corliss (1980) and Kaufman \& Sugar (1988). A limited number of oscillator strength values with given
Table 1. List of configurations of Sc ions generating fine-structure levels below the first ionization threshold.

\begin{tabular}{|c|c|c|c|c|c|}
\hline Ion & $n_{\mathrm{R}}$ & $n_{\mathrm{S}}$ & \multicolumn{3}{|c|}{ Configurations } \\
\hline Sc XXI & 55 & & $n l$ & & \\
\hline $\operatorname{Sc} x x$ & 55 & & $1 s^{2}$ & $1 \mathrm{~s} n l$ & \\
\hline Sc XIX & 54 & & $1 \mathrm{~s}^{2} n l$ & & \\
\hline \multirow[t]{2}{*}{ Sc XVIII } & 54 & 53 & $2 s^{2}$ & $2 \mathrm{~s} n l$ & \\
\hline & & & $2 \mathrm{p}^{2}$ & $2 \mathrm{p} n l$ & $(\leq 10)$ \\
\hline \multirow[t]{3}{*}{ Sc xVII } & 53 & 72 & $2 s^{2} n l$ & & \\
\hline & & & $2 \mathrm{~s} 2 \mathrm{p}^{2}$ & $2 \mathrm{~s} 2 \mathrm{p} n l$ & $(\leq 10)$ \\
\hline & & & $2 p^{3}$ & $2 \mathrm{p}^{2} n l$ & $(\leq 6)$ \\
\hline \multirow[t]{3}{*}{$\operatorname{SexvI}$} & 53 & 56 & $2 s^{2} 2 p^{2}$ & $2 \mathrm{~s}^{2} 2 \mathrm{p} n l$ & \\
\hline & & & $2 s 2 p^{3}$ & $2 \mathrm{~s} 2 \mathrm{p}^{2} n l$ & $(\leq 9)$ \\
\hline & & & $2 p^{4}$ & $2 \mathrm{p}^{3} n l$ & $(\leq 5)$ \\
\hline \multirow[t]{3}{*}{$\operatorname{Sc} x v$} & 53 & 51 & $2 s^{2} 2 p^{3}$ & $2 \mathrm{~s}^{2} 2 \mathrm{p}^{2} n l$ & \\
\hline & & & $2 s 2 p^{4}$ & $2 \mathrm{~s} 2 \mathrm{p}^{3} n l$ & $(\leq 9)$ \\
\hline & & & $2 \mathrm{p}^{5}$ & $2 \mathrm{p}^{4} n l$ & $(\leq 4)$ \\
\hline \multirow[t]{3}{*}{ Sc xIV } & 53 & 25 & $2 s^{2} 2 p^{4}$ & $2 \mathrm{~s}^{2} 2 \mathrm{p}^{3} n l$ & \\
\hline & & & $2 \mathrm{~s} 2 \mathrm{p}^{5}$ & $2 \mathrm{~s} 2 \mathrm{p}^{4} n l$ & $(\leq 6)$ \\
\hline & & & $2 p^{6}$ & $2 \mathrm{p}^{5} n l$ & $(\leq 4 p)$ \\
\hline \multirow[t]{3}{*}{ Sc XIII } & 53 & 16 & $2 s^{2} 2 p^{5}$ & $2 \mathrm{~s}^{2} 2 \mathrm{p}^{4} n l$ & \\
\hline & & & $2 s 2 p^{6}$ & $2 \mathrm{~s} 2 \mathrm{p}^{5} n l$ & $(\leq 5)$ \\
\hline & & & & $2 \mathrm{p}^{6} n l$ & $(\leq 3)$ \\
\hline \multirow[t]{2}{*}{ Sc XII } & 53 & 7 & $2 s^{2} 2 p^{6}$ & $2 \mathrm{~s}^{2} 2 \mathrm{p}^{5} n l$ & \\
\hline & & & & $2 \mathrm{~s} 2 \mathrm{p}^{6} n l$ & $(\leq 4)$ \\
\hline Sc XI & 52 & & $2 \mathrm{~s}^{2} 2 \mathrm{p}^{6} n l$ & & \\
\hline \multirow[t]{3}{*}{$\operatorname{Scx}$} & 52 & 32 & $3 s^{2}$ & $3 \mathrm{~s} n l$ & \\
\hline & & & $3 p^{2}$ & $3 \mathrm{p} n l$ & $(\leq 7)$ \\
\hline & & & $3 d^{2}$ & $3 \mathrm{~d} n l$ & $(\leq 5 \mathrm{~d})$ \\
\hline \multirow[t]{6}{*}{ Sc IX } & 51 & 42 & $3 \mathrm{~s}^{2} n l$ & & \\
\hline & & & $3 s 3 p^{2}$ & $3 \mathrm{~s} 3 \mathrm{p} n l$ & $(\leq 7)$ \\
\hline & & & $3 p^{3}$ & $3 \mathrm{p}^{2} n l$ & $(\leq 5 \mathrm{~d})$ \\
\hline & & & $3 \mathrm{~s} 3 \mathrm{~d}^{2}$ & $3 \mathrm{~s} 3 \mathrm{~d} n l$ & $(\leq 4)$ \\
\hline & & & $3 p 3 d^{2}$ & $3 \mathrm{p} 3 \mathrm{~d} n l$ & $(\leq 4 p)$ \\
\hline & & & $3 d^{3}$ & & \\
\hline \multirow[t]{7}{*}{ Sc VIII } & 51 & 41 & $3 s^{2} 3 p^{2}$ & $3 \mathrm{~s}^{2} 3 \mathrm{p} n l$ & \\
\hline & & & $3 \mathrm{~s} 3 \mathrm{p}^{3}$ & $3 \mathrm{~s} 3 \mathrm{p}^{2} n l$ & $(\leq 7 \mathrm{~d})$ \\
\hline & & & $3 s^{2} 3 d^{2}$ & $3 \mathrm{~s}^{2} 3 \mathrm{~d} n l$ & $(\leq 5 p)$ \\
\hline & & & $3 p^{4}$ & $3 \mathrm{p}^{3} n l$ & $(\leq 4)$ \\
\hline & & & $3 \mathrm{~s} 3 \mathrm{p} 3 \mathrm{~d}^{2}$ & $3 \mathrm{~s} 3 \mathrm{p} 3 \mathrm{~d} n l$ & $(\leq 4)$ \\
\hline & & & $3 p^{2} 3 d^{2}$ & $3 \mathrm{~s} 3 \mathrm{~d}^{3}$ & \\
\hline & & & $3 p 3 d^{3}$ & & \\
\hline \multirow[t]{7}{*}{ Sc VII } & 51 & 32 & $3 s^{2} 3 p^{3}$ & $3 \mathrm{~s}^{2} 3 \mathrm{p}^{2} n l$ & \\
\hline & & & $3 s 3 p^{4}$ & $3 \mathrm{~s} 3 \mathrm{p}^{3} n l$ & $(\leq 6)$ \\
\hline & & & $3 s^{2} 3 p 3 d^{2}$ & $3 \mathrm{~s}^{2} 3 \mathrm{p} 3 \mathrm{~d} n l$ & $(\leq 4)$ \\
\hline & & & $3 p^{5}$ & $3 \mathrm{p}^{4} n l$ & $(\leq 4 p)$ \\
\hline & & & $3 \mathrm{~s} 3 \mathrm{p}^{2} 3 \mathrm{~d}^{2}$ & $3 \mathrm{~s} 3 \mathrm{p}^{2} 3 \mathrm{~d} n l$ & $(\leq 4 p)$ \\
\hline & & & $3 \mathrm{~s}^{2} 3 \mathrm{~d}^{3}$ & $3 p^{3} 3 d^{2}$ & \\
\hline & & & $3 \mathrm{~s} 3 \mathrm{p} 3 \mathrm{~d}^{3}$ & & \\
\hline \multirow[t]{5}{*}{ Sc vI } & 51 & 16 & $3 s^{2} 3 p^{4}$ & $3 \mathrm{~s}^{2} 3 \mathrm{p}^{3} n l$ & \\
\hline & & & $3 s 3 p^{5}$ & $3 \mathrm{~s} 3 \mathrm{p}^{4} n l$ & $(\leq 5 p)$ \\
\hline & & & $3 s^{2} 3 p^{2} 3 d^{2}$ & $3 \mathrm{~s}^{2} 3 \mathrm{p}^{2} 3 \mathrm{~d} n$ & $(\leq 4 \mathrm{~d})$ \\
\hline & & & $3 p^{6}$ & $3 p^{5} 3 d$ & \\
\hline & & & $3 \mathrm{~s} 3 \mathrm{p}^{3} 3 \mathrm{~d}^{2}$ & $3 s^{2} 3 p 3 d^{3}$ & \\
\hline
\end{tabular}

1 Available: http://physics.nist.gov/asd 
Table 1. continued.

\begin{tabular}{|c|c|c|c|c|}
\hline Ion & $n_{\mathrm{R}}$ & $n_{\mathrm{S}}$ & \multicolumn{2}{|c|}{ Configurations } \\
\hline $\mathrm{Sc} \mathrm{v}$ & 51 & 10 & $\begin{array}{l}3 s^{2} 3 p^{5} \\
3 s^{3} 3 p^{6} \\
3 s^{2} 3 p^{3} 3 d^{2} \\
3 p^{6} 3 d\end{array}$ & $\begin{array}{l}3 \mathrm{~s}^{2} 3 \mathrm{p}^{4} n l \\
3 \mathrm{~s} 3 \mathrm{p}^{5} n l \quad(\leq 4 \mathrm{~d}) \\
3 \mathrm{~s}^{2} 3 \mathrm{p}^{3} 3 \mathrm{~d} n l(\leq 4 \mathrm{p}) \\
3 \mathrm{~s} 3 \mathrm{p}^{4} 3 \mathrm{~d}^{2}\end{array}$ \\
\hline Sc IV & 51 & 3 & $\begin{array}{l}3 s^{2} 3 p^{6} \\
3 s^{3} 3 p^{6} 3 d \\
3 s^{2} 3 p^{4} 3 d^{2}\end{array}$ & $\begin{array}{l}3 s^{2} 3 p^{5} n l \\
3 s^{3} 3 p^{6} 4 s\end{array}$ \\
\hline Sc III & 50 & & $3 \mathrm{~s}^{2} 3 \mathrm{p}^{6} n l$ & \\
\hline
\end{tabular}

Notes. $n_{\mathrm{R}}$ is the number of configurations of the Rydberg series built on the ground configuration of the parent ion, which is limited to shells up to $n=10$. In addition $n_{\mathrm{S}}$ is the number of core-excited configurations. The notation $n l(\leq 6)($ resp. $n l(\leq 5 \mathrm{p}))$ means that shells up to $n=6$ (resp. sub-shells up to $n l=5 \mathrm{p}$ ) are included in the series. Levels with higher subshells lie above the limit.

accuracy can also be found. They were supplemented with the $g f$-values computed by Kurucz for Sc III to Sc IX, which are based on the same energy levels as in the NIST compilation. Papers that have adressed scandium include Wilson et al. (2000) (Sc v) and Sossah et al. (2008) (Sc III and Sc IV). We first examine the energy structure.

\subsection{Correspondence between FAC and NIST levels}

Our results for the electronic ground states given in Table 2 agree with those of NIST in terms of the configuration and the total angular momentum. We took the term assignments ${ }^{2 S+1} L$ from NIST because FAC uses $j j$-coupling. The ionization energies for the nineteen ions are compared in this same table. The difference from FAC amounts to a few tenths of percent for Sc xxI to $\mathrm{Sc} v$ reaching a maximum of $0.5 \%$ for the closed shell ion Sc xII, an exception being Sc vII (with 1.2\%). The relative difference is $1.7 \%$ for the other closed shell ion Sc IV, and for the least ionized Sc III it reaches $3.9 \%$. We note that the NIST ionization energy uncertainties derived by Sugar \& Corliss (1980) amount to a few units in the last digit of the quoted value, and are often of the order of the differences with FAC. Our results compare favourably with the systematic calculations of Rodrigues et al. (2004), which are reported in the last columns of Table 2, in particular for Sc XVIII and the lower ionization stages. However, in Rodrigues et al. (2004) almost all the periodic table was investigated, precluding any peculiar improvements, while we performed some adjustments in choosing the levels to minimize on.

The NIST database contains 820 levels ${ }^{2}$. They are distributed among ions according to the second column of Table 2 with $30 \%$ of them being in Sc v and Sc IV. Our large-scale computation leads to much more levels (exactly 21842 below the first thresholds, for a total of 28928), with the largest number of levels corresponding to ions with nearly half-filled $2 p$ or $3 p$ sub-shells in their ground state (third column). The reason for such a large number is somewhat different for the two cases, as can be seen by comparing the structure of Sc XV and Sc vII in Table 1. In addition to the Rydberg series converging to the first threshold, the

\footnotetext{
2 The fake level $2 s^{2} 2 p^{2}\left({ }^{1} \mathrm{P}\right) 3 \mathrm{~d}^{2} \mathrm{P}_{3 / 2}$ of $\mathrm{Sc} \mathrm{xv}$ was discarded, as well as the level $3 s 3 p^{6} 5 p^{1} P_{1}^{\circ}$ of $\mathrm{Sc}$ iv and seven levels from the configurations $1 \mathrm{~s} 2 \mathrm{~s} 2 \mathrm{p}$ and $1 \mathrm{~s} 2 \mathrm{p}^{2}$ of Sc XIx, because they are above the ionization threshold.
}

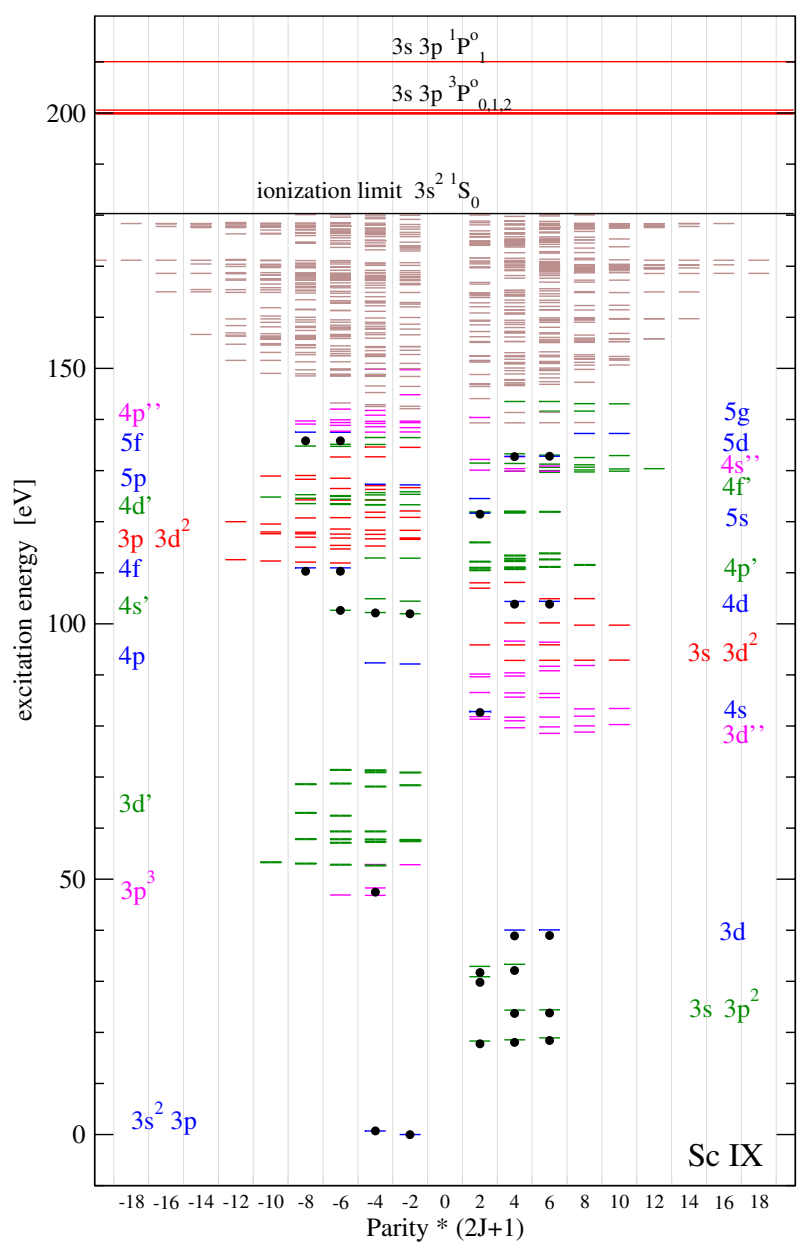

Fig. 1. Energy levels below the ionization threshold for Sc IX, calculated by FAC. Abscissa is the total angular momentum degeneracy $2 J+1$ multiplied by $+1 /-1$ for even/odd levels, respectively. Black dots represent the levels from the NIST database. The lowest levels configurations are named according to their parent core: $n l$ means $3 s^{2} n l$ (blue); $n l^{\prime}$ means $3 \mathrm{~s} 3 \mathrm{p} n l$ (green); $n l^{\prime \prime}$ means $3 \mathrm{p}^{2} n l$ (magenta). First ionization thresholds (levels of Sc $\mathrm{x}$ ) are also shown.

most ionized $\mathrm{Sc} \mathrm{xv}$ has a series for which the external electron extends to the $n=9$ shell. In Sc viI, the external electron remains below $n=6$ but many complex configurations are permitted by exciting electrons from $3 \mathrm{~s}$ or $3 p$ to $3 d$.

A principal objective of this work was to map the 820 NIST levels to FAC ones so as to compare the energy spectra and evaluate the accuracy of the calculated energies. The natural approach is to match their configuration designation and their exact symmetries - total momentum $J$ and parity. However, these lone parameters do not ensure uniqueness. The classification in NIST utilizes $L S$-coupling, which is meaningful for most of the levels of scandium. FAC uses $j j$-coupling, thus the term designation, particularly for the parent core of a configuration, is not of direct help. We plotted in Fig. 1 the Sc Ix levels obtained from our calculations and from NIST. This case, which is not particularly difficult (26 levels in NIST), will be used as an illustration in the following general discussion.

As long as a given configuration is complete in NIST, there is no ambiguity in assigning the levels. Indeed the results of FAC confidently reproduce fine structure splitting, such that a global energy shift of the configuration suffices. This situation occurs generally for the ground configurations and the first few excited ones, obviously the most commonly observed experimentally, 
Table 2. Number of levels, ground states, and ionization energies (in $\mathrm{eV}$ ) for the nineteen ions of scandium according to NIST and our calculations (FAC).

\begin{tabular}{|c|c|c|c|c|c|c|c|c|c|}
\hline \multirow[b]{2}{*}{ Ion } & \multicolumn{2}{|c|}{ Number of levels } & \multicolumn{2}{|c|}{ Ground state } & \multicolumn{5}{|c|}{ Ionization energy $(\mathrm{eV})$} \\
\hline & NIST & FAC & Conf & Level & NIST & FAC & Diff & Rodrigues & Diff \\
\hline Sc XXI & 25 & 100 & $1 \mathrm{~s}$ & ${ }^{2} S_{1 / 2}$ & 6033.755 & 6033.82 & 0.06 & & \\
\hline Sc $x x$ & 25 & 199 & $1 s^{2}$ & ${ }^{1} \mathrm{~S}_{0}$ & 5675. & 5673.45 & -1.55 & & \\
\hline Sc XIX & 27 & 99 & $2 \mathrm{~s}$ & ${ }^{2} \mathrm{~S}_{1 / 2}$ & 1288.0 & 1287.61 & -0.39 & & \\
\hline Sc XVIII & 16 & 676 & $2 s^{2}$ & ${ }^{1} \mathrm{~S}_{0}$ & 1212.6 & 1212.90 & 0.30 & 1206.9 & -5.70 \\
\hline Sc xVII & 28 & 1332 & $2 s^{2} 2 p$ & ${ }^{2} \mathrm{P}_{1 / 2}^{\circ}$ & 1094. & 1092.73 & -1.27 & 1094.8 & 0.80 \\
\hline Sc xVI & 34 & 2298 & $2 s^{2} 2 p^{2}$ & ${ }^{3} \mathrm{P}_{0}$ & 1009. & 1008.91 & -0.09 & 1010.0 & 1.00 \\
\hline Sc $x v$ & 27 & 2577 & $2 s^{2} 2 p^{3}$ & ${ }^{4} \mathrm{~S}_{3 / 2}^{\circ}$ & 927.5 & 925.69 & -1.81 & 926.3 & -1.20 \\
\hline Sc XIV & 35 & 2286 & $2 s^{2} 2 p^{4}$ & ${ }^{3} \mathrm{P}_{2}$ & 830.8 & 831.67 & 0.87 & 830.7 & -0.10 \\
\hline Sc XIII & 29 & 1476 & $2 s^{2} 2 p^{5}$ & ${ }^{2} \mathrm{P}_{3 / 2}^{\circ}$ & 756.7 & 755.97 & -0.73 & 755.8 & -0.90 \\
\hline Sc XII & 55 & 569 & $2 s^{2} 2 p^{6}$ & ${ }^{1} \mathrm{~S}_{0}^{3 / 2}$ & 687.4 & 684.14 & -3.22 & 684.5 & -2.86 \\
\hline $\mathrm{Sc} \mathrm{XI}$ & 39 & 96 & $3 \mathrm{~s}$ & ${ }^{2} \mathrm{~S}_{1 / 2}$ & 249.80 & 249.41 & -0.39 & 249.2 & -0.60 \\
\hline Sc $x$ & 67 & 523 & $3 s^{2}$ & ${ }^{1} \mathrm{~S}_{0}$ & 225.18 & 224.89 & -0.29 & 222.9 & -2.28 \\
\hline Sc IX & 26 & 915 & $3 s^{2} 3 p$ & ${ }^{2} \mathrm{P}_{1 / 2}^{\circ}$ & 180.0 & 180.31 & 0.31 & 179.6 & -0.40 \\
\hline Sc VIII & 26 & 2001 & $3 s^{2} 3 p^{2}$ & ${ }^{3} \mathrm{P}_{0}$ & 158.1 & 157.80 & -0.30 & 157.4 & -0.70 \\
\hline Sc VII & 34 & 2661 & $3 s^{2} 3 p^{3}$ & ${ }^{4} \mathrm{~S}_{3 / 2}^{\circ}$ & 138.0 & 136.33 & -1.67 & 135.8 & -2.20 \\
\hline $\mathrm{Sc}$ VI & 39 & 1889 & $3 s^{2} 3 p^{4}$ & ${ }^{3} \mathrm{P}_{2}$ & 110.7 & 110.07 & -0.63 & 109.3 & -1.40 \\
\hline $\mathrm{Sc} v$ & 118 & 1436 & $3 s^{2} 3 p^{5}$ & ${ }^{2} \mathrm{P}_{3 / 2}^{\circ}$ & 91.7 & 91.42 & -0.27 & 90.3 & -1.39 \\
\hline Sc IV & 127 & 616 & $3 s^{2} 3 p^{6}$ & ${ }^{1} \mathrm{~S}_{0}$ & 73.4894 & 74.76 & 1.27 & 72.3 & -1.19 \\
\hline Sc III & 43 & 93 & $3 p^{6} 3 d$ & ${ }^{2} \mathrm{D}_{3 / 2}$ & 24.75684 & 23.38 & -1.37 & 23.8 & -0.96 \\
\hline total & 820 & 21842 & & & . & & & & \\
\hline
\end{tabular}

Notes. Ionization energies derived from Rodrigues et al. (2004) are also given. Columns "diff" give the differences between our calculations or Rodrigues' ones and NIST values.

such as $3 s^{2} 3 p, 3 s 3 p^{2}$, and $3 s^{2} 3 d$ in Sc IX (Fig. 1). It also occurs for some high lying configurations, which are perhaps embedded in a dense energy pattern, but with a simple structure (such as $3 \mathrm{~s}^{2} 4 \mathrm{~s}, 4 \mathrm{~d}, 4 \mathrm{f}, 5 \mathrm{~s}, 5 \mathrm{~d}, 5 \mathrm{f}$ in Sc $\mathrm{Ix}$; note that $4 \mathrm{p}$ and $5 \mathrm{p}$ are missing in NIST).

For some configurations that are incomplete in NIST, the correspondence is obvious thanks again to the fidelity of FAC in reproducing the fine-structure energy pattern. Examples are the three levels out of seven of the configuration 3s $3 \mathrm{p} 4 \mathrm{~s}$ (labelled $4 \mathrm{~s}$ ' in Fig. 1). In some complex cases, we used the original literature. Figure 2 shows the example of the $2 s^{2} 2 p^{3} 3 d$ configuration in Sc xIV with its 38 fine structure levels (FAC) and 18 experimental levels (NIST, black dots). For the $J=2$ and $J=3$ symmetries, there is some ambiguity in assigning the third from the top NIST levels. This ambiguity is removed when referring to the data in Bromage \& Fawcett (1977c, red diamonds). We note the quality of the theoretical FAC results: when shifted down by $0.55 \mathrm{eV}$ (one thousandth of the excitation energy), the standard deviation from the experimental data (Bromage \& Fawcett 1977c) is $0.32 \mathrm{eV}$, which should be compared with a spread of $37 \mathrm{eV}$ for the whole configuration and a distance of $0.48 \mathrm{eV}$ for the closest levels.

When several configurations lead to levels of the same symmetries in a narrow energy range, the situation becomes more difficult as one might expect for a configuration interaction. For highly ionized ions, this generally involves simple configurations and the correspondence is often evident. For the least ionized ions (14 to 18 electrons), the rich configuration $3 \mathrm{~s}^{2} 3 \mathrm{p}^{n-1} 3 \mathrm{~d}$ often interacts with the $3 \mathrm{~s}^{2} 3 \mathrm{p}^{n-1} 4 \mathrm{~s}$ or $3 \mathrm{~s} 3 \mathrm{p}^{n+1}$ configurations. These configurations are connected through one-electron jump to the ground state $3 s^{2} 3 p^{n}$, thus they have been partially observed and recorded in NIST. The mixing of $3 s^{2} 3 \mathrm{p}^{n-1} 4 \mathrm{p}$ with $3 \mathrm{~s} 3 \mathrm{p}^{n} 3 \mathrm{~d}$ and $3 s^{2} 3 p^{n-2} 3 d^{2}$ is still more intricate, but this group of levels is only observed in Sc v and Sc IV. We had to carefully check these

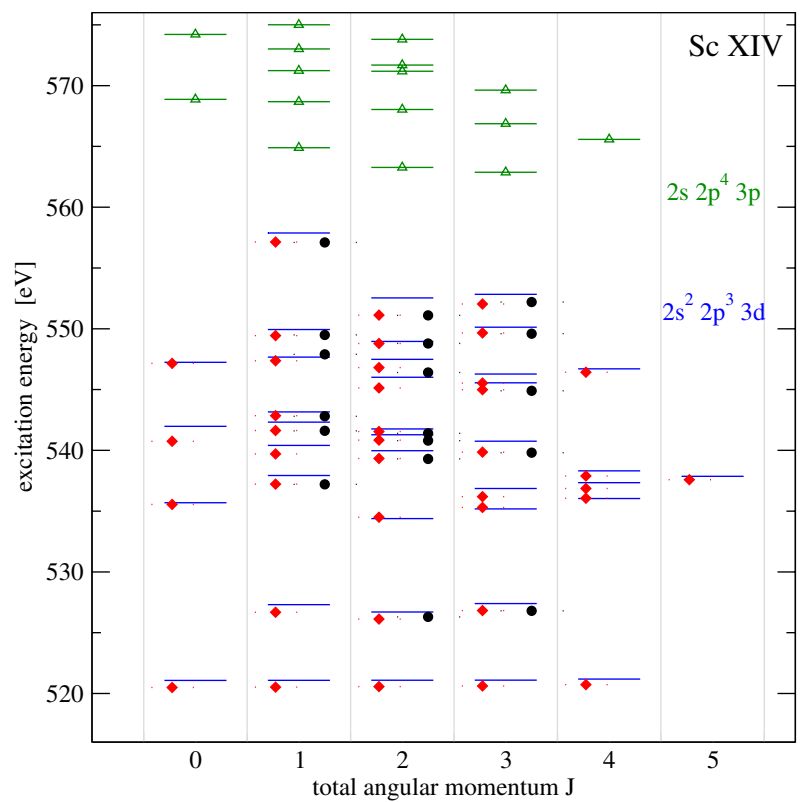

Fig. 2. Comparison of the energy levels of the $2 s^{2} 2 p^{3} 3 d$ in Sc xIv configuration. Blue: as calculated by FAC; black dots: from the NIST database; red diamonds: from the original paper Bromage \& Fawcett (1977c). Also shown (green, triangles) are the close levels from the $2 \mathrm{~s} 2 \mathrm{p}^{4} 3 \mathrm{p}$ configuration.

levels when assessing the assignement. In a few cases ${ }^{3}$, we had to use the transition data to discriminate between several candidates.

\footnotetext{
${ }^{3}$ Namely for some $3 p^{5} 6 \mathrm{~g}(J=4,5)$ levels in Sc Iv, and $3 \mathrm{p}^{3} 3 \mathrm{~d}$ and $3 \mathrm{p}^{3} 4 \mathrm{~d}(J=2,3)$ levels in Sc vi.
} 
We were finally able to establish a correspondence for all the levels contained in the NIST database. For most of them, the NIST designation was confirmed. A very few states are highly mixed and the FAC dominant contribution appears to differ from the NIST one. This is unimportant in as much as the level energies and the transition probabilities are correctly reproduced. More details and comments concerning the electronic structure of individual scandium ions may be found in the appendix.

\subsection{Comparison of theoretical and experimental energies and adjustment}

The differences in the level excitation energies between NIST and our results are plotted in Fig. 3 as a function of the NIST excitation energies. Differences in ionization energies are also drawn. The accuracy ${ }^{4}$ reported by NIST for its energy values is most often below $0.1 \mathrm{eV}$ and at most $1 \mathrm{eV}$ for a very few levels, hence irrelevant in this figure. Though these panels show all NIST levels, they contain fewer than $4 \%$ of the FAC levels (see last line of Table 2). The NIST databank is complete only for the lowest energy range of an ion electronic structure. Most of the excited states are embedded in a background of levels that are missing from the database (Fig. 1 is a clear illustration for the Sc Ix case).

The vertical scale in Fig. 3 is the same in all the panels, while the abscissa is roughly adjusted to the ionization energies. As expected, the global trend is a decrease in accuracy as one goes from the highly ionized ions to the near-to-neutral ones, when the nucleus attraction no longer dominates electron-electron interactions. Our excitation energies are usually above NIST ones with the exception of Sc xx, Sc xII, Sc XI, and Sc III. These ions have a simple ground state, either with closed shells $\left(1 \mathrm{~s}^{2}\right.$ and $2 s^{2} 2 p^{6}$ ) or one electron above closed shells ( $3 s$ and $3 s^{2} 3 p^{6} 3 d$ ). For these ions and some others (notably Sc IV), the ionization energy difference is consistent with an extrapolation from excited levels. This is certainly not the case for Sc XVII, Sc Xv, and Sc VII. The NIST ionization energies are extrapolated from bound level series, which stop far from the limit for these ions. Moreover, these ions have several ionization thresholds close to their ionization limit. As the FAC series are consistent by construction (the first series converges to the limit), it is tempting to attribute the inconsistency in ionization energy differences to a difficulty of NIST in determining the correct limit. The accuracy for the ionization potentials given in Kaufman \& Sugar (1988) is less than $2000 \mathrm{~cm}^{-1}(0.2 \mathrm{eV})$ for Sc III to Sc XII, and increases from $12000 \mathrm{~cm}^{-1}$ to $20000 \mathrm{~cm}^{-1}$ (from $1.5 \mathrm{eV}$ to $2.5 \mathrm{eV}$ ) for ions Sc XIII to Sc XVIII. This makes the differences in ionization energies between FAC and NIST much less critical for this last series of ions, and in particular for Sc XVII and Sc Xv.

Excitation energies assume that the ground state is the reference level. An error in the ground state absolute energy would result in a shift in all the excitation energies. To judge the quality of our results, we computed the variance, i.e. the rms of the deviation between the differences in excitation energies and their mean value (when given in relative values, the variance is relative to the ionization energy). This quantifies the extent to which the FAC and NIST energy spectra can be adjusted by shifting one of them relative to the other as a block.

For the H-like ion Sc xxI, the agreement is excellent with a variance of $12 \mathrm{meV}\left(100 \mathrm{~cm}^{-1}\right)$. This is almost entirely due to the ground state. When the latter is raised by $0.063 \mathrm{eV}$, the variance

\footnotetext{
4 This accuracy is determined from the number of significant digits in the NIST data values.
}

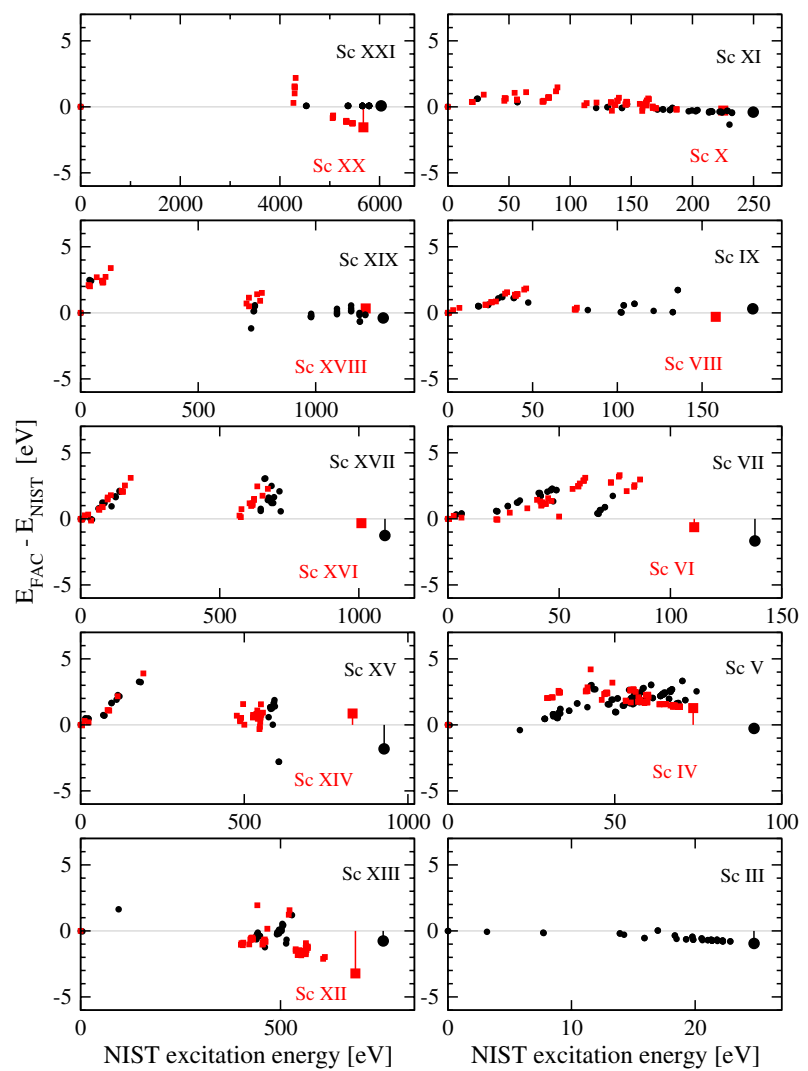

Fig. 3. Excitation energy differences (in eV) between our results and NIST data for all the levels of the nineteen scandium ions in the NIST database, as a function of the NIST excitation energy (in eV). The differences between ionization energies are also plotted as a larger symbol with a drop line to the zero axis and the ion name nearby.

becomes $1.8 \mathrm{meV}\left(14 \mathrm{~cm}^{-1}\right)$. For comparison, the $2 \mathrm{~s}_{1 / 2}-2 \mathrm{p}_{1 / 2}$ splitting is $2200 . \mathrm{cm}^{-1}$. We note that NIST energies are also theoretical for this ion. The variance is smaller than $0.15 \%$ from Sc xx to Sc x. Systematic discrepancies appear for the lowest levels of L-shell ions Sc XIX to Sc XIII with ground configurations of the type $1 s^{2} 2 l^{q}, q=1$ to 7 . These levels (below $200 \mathrm{eV}$ in the left column of Fig. 1) include the excitation of one or two of the electrons $2 \mathrm{~s}$ to $2 \mathrm{p}$. They are systematically calculated to be too high by a few $\mathrm{eV}$, the difference increasing with energy. The FAC code uses a single adjusted potential to generate one electron wavefunctions, which might explain the difficulty in representing at the same time the ground configuration and the core-excited ones. Though it is possible to slightly improve the accuracy by playing with the configurations to be minimized, obtaining results accurate to a few tenths of $\mathrm{eV}$ would require far more sophisticated approaches. For instance, Gu (2005) supplemented configuration interaction calculations with a many-body perturbation theory (MBPT) to perform a systematic study of the previous levels in L-shell ions.

The variance is smaller than $0.5 \%$ for Sc IX to Sc vII, smaller than $1 \%$ for Sc vi to Sc IV, and $1.5 \%$ for Sc III. The calculation encounters some difficulties in precisely determining the level energies of Sc VII to Sc IV of medium ionization charge and with the ground states of the form $3 \mathrm{~s}^{2} 3 \mathrm{p}^{q}, q=3$ to 6 . In addition to the aforementioned reasons, these cases contain a number of large configuration interactions, as can be verified from the output of the code. For example, in Sc vi the $J=1$ levels of the even configurations $3 \mathrm{~s} 3 \mathrm{p}^{4} 3 \mathrm{~d}$ and $3 \mathrm{~s}^{2} 3 \mathrm{p}^{2} 3 \mathrm{~d}^{2}$ are mixed with maximum leading percentages lower than $40 \%$. 
Table 3. Number of transitions in scandium spectra (first column) available from three sources: NIST database, Kurucz linelist (CD-ROM No. 23) and present calculations with FAC.

\begin{tabular}{|c|c|c|c|c|c|c|c|c|c|}
\hline \multirow[b]{2}{*}{ Ion } & \multirow[b]{2}{*}{ Total $^{a}$} & \multicolumn{2}{|c|}{ NIST E1 transitions } & \multirow[b]{2}{*}{$\begin{array}{l}\text { only } \\
\text { obs }\end{array}$} & \multirow[t]{2}{*}{ Kurucz } & \multicolumn{4}{|c|}{ FAC E1 transitions } \\
\hline & & $\begin{array}{c}\text { obs } \\
\text { with } g f\end{array}$ & $\begin{array}{c}\text { Ritz } \\
\text { with } g f\end{array}$ & & & $\begin{array}{c} \\
g f \\
>10^{-4} \\
\end{array}$ & $\begin{array}{r} \\
g f \\
>10^{-3} \\
\end{array}$ & $\begin{array}{c}g A / g A_{\max } \\
\quad>10^{-5}\end{array}$ & $\begin{array}{c}g f \mathrm{e}^{-5 E / I} \\
>10^{-4}\end{array}$ \\
\hline $\mathrm{Sc} \mathrm{xxI}^{b}$ & 8 & 0 & 0 & 0 & & 780 & 732 & 606 & 588 \\
\hline Sc $x x$ & 37 & 0 & 37 & 0 & & 2549 & 2194 & 1350 & 1493 \\
\hline Sc XIX & 79 & 29 & 47 & 3 & & 790 & 754 & 701 & 684 \\
\hline Sc XVIII & 24 & 16 & 8 & 0 & & 21505 & 13999 & 10681 & 9449 \\
\hline Sc xvII & 52 & 21 & 20 & 11 & & 72398 & 39927 & 26906 & 23070 \\
\hline Sc xvI & 82 & 34 & 30 & 18 & & 171420 & 90024 & 59607 & 49068 \\
\hline $\mathrm{Sc} x \mathrm{v}$ & 58 & 28 & 14 & 16 & & 216773 & 114497 & 68431 & 61169 \\
\hline Sc XIV & 63 & 33 & 21 & 9 & & 158608 & 85919 & 49826 & 46103 \\
\hline Sc XIII & 39 & 18 & 1 & 20 & & 74152 & 43995 & 28290 & 25346 \\
\hline Sc XII & 68 & 5 & 0 & 63 & & 16007 & 11620 & 8084 & 7606 \\
\hline Sc XI & 130 & 31 & 86 & 13 & & 763 & 746 & 719 & 714 \\
\hline $\operatorname{Sc} x$ & 99 & 25 & 4 & 70 & & 20974 & 15774 & 14387 & 11723 \\
\hline Sc IX & 35 & 15 & 0 & 20 & 95 & 67043 & 43752 & 39634 & 29692 \\
\hline Sc VIII & 46 & 16 & 3 & 27 & 72 & 260142 & 155366 & 143356 & 95592 \\
\hline Sc VII & 53 & 15 & 5 & 33 & 124 & 408929 & 236975 & 207634 & 133874 \\
\hline Sc vI & 63 & 0 & 0 & 63 & 119 & 191222 & 112340 & 92957 & 65824 \\
\hline $\mathrm{Sc} v$ & 454 & 14 & 0 & 440 & 36 & 104336 & 63866 & 36519 & 35989 \\
\hline Sc IV & 407 & 4 & 0 & 403 & 1204 & 19657 & 13802 & 5921 & 8691 \\
\hline $\mathrm{Sc}_{\text {III }}$ & 133 & 79 & 18 & 36 & 226 & 735 & 682 & 656 & 615 \\
\hline total & 1930 & 383 & 294 & 1245 & 1876 & 1808783 & 1046964 & 796265 & 607290 \\
\hline
\end{tabular}

Notes. The total number of dipolar electric transitions found in NIST (second column) is broken down into observed transitions given with $g f$ values (third column), Ritz unobserved transitions with $g f$ values (fourth column), and observed transitions without $g f$ values (fifth column). All transitions listed by Kurucz have $g f$ values (sixth column). The four last columns give the number of transitions calculated in this work and selected according to various criteria: lines with $g f$ values greater than $10^{-4}$ and $10^{-3}$, lines with weighted transition probability $g A$ greater than $10^{-5}$ relative to the maximum value in the ion. Finally (last column), transitions have been selected according to their $g f$ values weighted by a Bolztmann factor $\mathrm{e}^{-E / k T}$, where $E$ is the lower level excitation energy and the energy $k T$ is one fifth of the ionization potential of the ion. (a) Excluding 9 transitions in Sc xIX and 1 transition in Sc Iv from levels above the ionization threshold, and 4 observed lines in Sc vi without level identification. ${ }^{(b)}$ NIST contains only theoretical wavelengths for this ion.

These comparisons with experimental data show that as a whole our results are of medium accuracy when compared to high precision atomic studies found in the literature (see for instance Hibbert et al. 2010, and the associated Special Issue on High precision atomic physics). The latter are however tailored to the cases under scrutiny and generally address a very limited number of levels. Our goal is in contrast to produce a large-scale database for scandium atomic structure that includes high lying levels as well and remains accurate, homogeneous, and consistent. The previous comparison with experimental data has shown that our results fullfill this aim.

To take advantage of our mapping between calculated results and NIST data, we performed some a posteriori adjustments to the FAC level energies. We found that it was unsatisfactory to simply set the excitation energies to the NIST values for all the states with a correspondence in NIST. As shown in Figs. 1-3, the corrections are minor for individual levels. However, for many excited configurations that are incomplete in NIST and surrounded with numerous predicted levels, it would be misleading to displace only a few levels. We adjusted to NIST values the levels of the lowest configurations that are complete, except for when the corresponding energy range includes other levels without any correspondence in NIST. Levels of higher energy were displaced as a few blocks (usually one or two) with an energy shift adjusted to compensate for the mean difference between the FAC and NIST energies. Some lower isolated but incomplete configurations were also individually adjusted as a block. Though somewhat arbitrary, this procedure preserves the FAC level splittings within incomplete configurations and allows us to obtain realistic patterns of predicted lines in individual multiplets. More details for each ion are given in the appendix. In any case, the data we deliver in the electronic tables allow one to go back to the original calculated values. Ionization energies were also adjusted for consistency.

\subsection{Comparison of theoretical and experimental oscillator strengths}

We used FAC to calculate in a second step the electric dipole transition data for the nineteen ionization stages. The FAC capabilities allow us to work in the complete configuration spaces described in Sect. 2. From a total number of transitions of around six million for all the ions, three and a half connect the levels below the limits. The ions $\mathrm{Sc} \mathrm{xv}$ and $\mathrm{Sc}$ vII have the richest spectra, which reflects their large number of levels (see Table 2). The ion Sc VII has eight hundred times more transitions than the ions with a closed core, most of them between high levels. A census of the lines according to various selection criteria is given in the last four columns of Table 3 . With a threshold of $10^{-4}$ for the weighted oscillator strength $g f$, the number of lines is divided by two (Col. 7). After increasing the threshold to $10^{-3}$, the number is further reduced by half (Col. 8). As the weighted transition probability $g A$ scales as $g f$ multiplied by the square of the transition energy, applying thresholds using $g A$ put transitions connecting high levels at a disavantage. Retaining lines with a transition probability smaller by five orders than the strongest one, one gets fewer transitions than with $g f>10^{-3}$ (Col. 9). A Bolztmann factor can also decrease the weight of high levels. With a factor mimicking a thermal population at a temperature equal to one fifth of the ionization potential, one third of the 


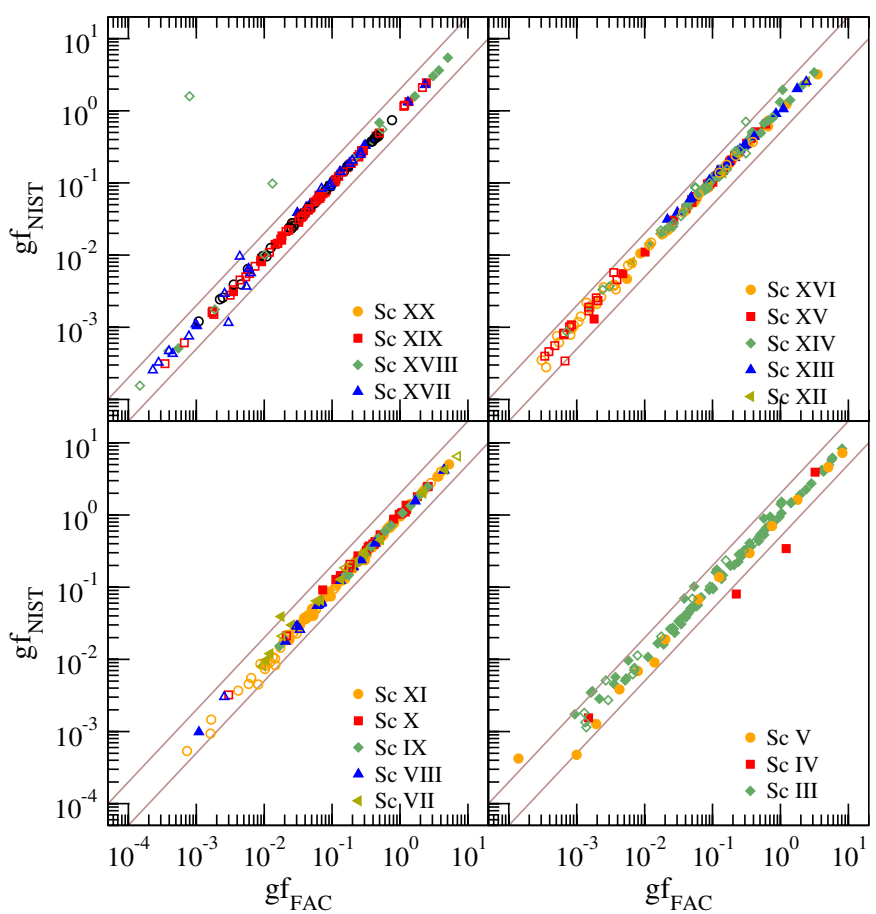

Fig. 4. Scandium weighted oscillator strengths $g f$ for the 677 transitions in the NIST database as a function of our calculated values. Filled and open symbols refer, respectively, to transitions that are observed, or not observed and predicted from two observed levels. NIST has no oscillator strength for Sc xxI and Sc vi. The two grey lines delineate the values $g f_{\mathrm{NIST}} / g f_{\mathrm{FAC}}=2$ or $1 / 2$.

transitions are retained than with the same threshold (compare Cols. 10 and 7).

As all the levels in NIST were identified within our level list, we were able to test the reliability of our results by comparing our transition data with all $^{5}$ the NIST transitions. The second to fifth columns in Table 3 describe the distribution of the transitions among ions in the NIST database, according to whether they are given with an oscillator strength value or not, and whether they are experimentally observed or deduced by Ritz predictions from two observed levels. The 677 transitions with $g f$ values represent $35 \%$ of a total of 1930 . A little more than half of them are observed. The ions Sc v and Sc IV contain $45 \%$ of the transitions, but only $4 \%$ of those have $g f$ values. Among the latter, some $17 \%$ belong to Sc XI, and most of them belong to the higher ionization stages with the exception of Sc III $(14 \%)$. This is likely due to their electronic structure being easier to analyse. Both Sc XXI and Sc vi have no oscillator strength in NIST.

In Fig. 4, we compare the oscillator strengths given by NIST with our results for all the studied ions. Observed transitions are marked as filled symbols, while open symbols denote the predicted Ritz transitions. The two lines delineate the limit of the range $0.5<g f_{\mathrm{NIST}} / g f_{\mathrm{FAC}}<2$. Most of the 677 transitions definitely fall between these lines, in particular the numerous transitions of Sc XI and Sc III. In view of the number of lines involved, this constitutes a reassuring indication of reliability. We emphasize that the connection between the NIST levels and our levels was based on symmetry and configuration characteristics, and used transition data for only a few levels (see footnote 3 ).

\footnotetext{
5 Excluding electric quadrupole and magnetic dipole transitions: their oscillator strengths are very low (a maximum of $\log g f=-4.8$ for a $3 \mathrm{~s}-3 \mathrm{~d}$ transition in $\mathrm{Sc} \mathrm{XIII})$.
}

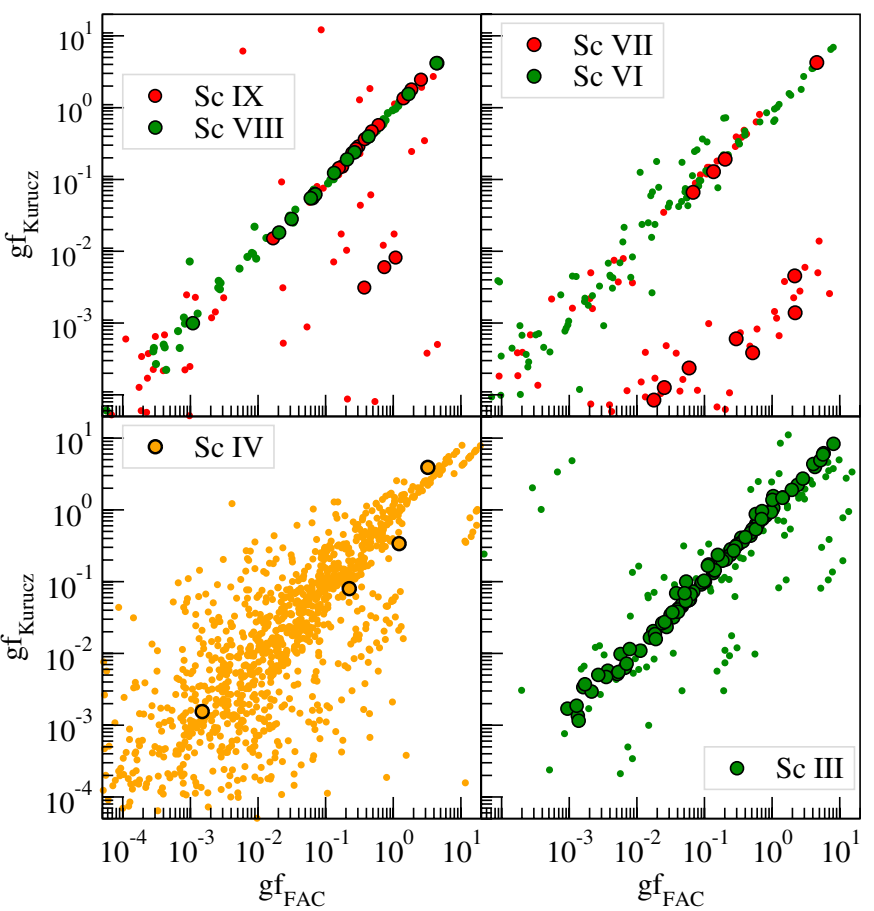

Fig. 5. Scandium weighted oscillator strengths $g f$ for the 1840 transitions in the Kurucz linelist (excluding $\mathrm{Sc} v$ ) as a function of our calculated values. Large symbols refer to the transitions that are also common to NIST.

Two transitions show divergent $g f$ values in Sc xvIII (upper left panel). We are confident that this is an error in NIST as those two lines imply a two-electron jump (see Appendix). In addition to the data for Sc XxI and Sc vi that have no oscillator strengths with which to compare, Sc IV is in desperately short supply of test data (lower right panel).

The Kurucz linelist ${ }^{6}$ for spectra Sc IX to Sc III provides a set of $g f$-values that are ten times larger than NIST (see sixth column in Table 3). Kurucz data, which is widely used in the stellar community, was the largest available source of data for scandium. They are based on the same set of levels as in NIST, hence the NIST wavelengths are reproduced in the Kurucz linelist. The Kurucz oscillator strengths are either duplicated from NIST, or independently computed. We excluded the Kurucz data for Sc v, which appeared obsolete (see Appendix). Figure 5 compares our calculated oscillator strengths with Kurucz ones. Any disagreements appear significantly larger than in Fig. 4, depending on the ion. For Sc VIII, Sc vI, and most of the lines in Sc III, the comparison is satisfactory. For the ions Sc IX, Sc vII, and Sc IV, there are large discrepancies. To trace the origin of these discrepancies, we plotted in the figure as bigger symbols the transitions that have a $g f$ value in NIST. From Fig. 4, we know that our values match NIST ones. It appears that Kurucz miscalculated the $g f$ value of a number of lines of Sc IX and Sc VII (upper panels). The problem seems linked to the quadruplet system in Sc IX and an inverted identification of some levels in Sc viI (see Appendix). In the case of Sc IV (lower left panel), it is difficult to draw any strong conclusion as only 4 transitions have $g f$ values in NIST. For Sc III, our calculations clearly match the $97 \mathrm{gf}$ values for transitions shared with NIST and we are confident in our results.

\footnotetext{
61995 Atomic Line Data (Kurucz and Bell) Kurucz CD-ROM No.23. Cambridge, Mass.: Smithsonian Astrophysical Observatory.
} 
Table 4. Excerpt of the table of lines restricted to $g f>10^{-3}$ (see text for explanations).

\begin{tabular}{|c|c|c|c|c|c|c|c|c|c|c|c|c|c|}
\hline $\begin{array}{c}\lambda \\
(\mathrm{nm})\end{array}$ & $\log g f$ & Ion code & $\begin{array}{c}E_{1} \\
\left(\mathrm{~cm}^{-1}\right)\end{array}$ & $J_{1}$ & $\begin{array}{c}E_{2} \\
\left(\mathrm{~cm}^{-1}\right)\end{array}$ & $J_{2}$ & $n l_{1}$ & $n l_{2}$ & Tag & $\begin{array}{c}\lambda_{\mathrm{N}} \\
(\mathrm{nm})\end{array}$ & $\log g f_{\mathrm{N}}$ & $\begin{array}{c}E_{1, \mathrm{~N}} \\
\left(\mathrm{~cm}^{-1}\right)\end{array}$ & $\begin{array}{c}E_{2, \mathrm{~N}} \\
\left(\mathrm{~cm}^{-1}\right)\end{array}$ \\
\hline 58.278 & -2.030 & 21.02 Sc III & 197.6 & 2.5 & 171787.6 & 2.5 & $3 d$ & $6 f$ & $\bar{P}$ & 58.2785 & & 197.64 & 171787.64 \\
\hline 58.278 & -0.727 & $21.02 \mathrm{Sc}$ III & 197.6 & 2.5 & 171787.6 & 3.5 & $3 d$ & $6 f$ & No & 58.2780 & & 197.64 & 171787.64 \\
\hline 58.932 & -2.975 & $21.02 \mathrm{Sc}$ III & 0.0 & 1.5 & 169685.9 & 1.5 & $3 d$ & $7 p$ & No & 58.9320 & & 0 . & 169685.9 \\
\hline 58.949 & -2.281 & $21.02 \mathrm{Sc}$ III & 0.0 & 1.5 & 169638.0 & 0.5 & $3 d$ & $7 p$ & No & 58.9490 & & 0 . & 169637.96 \\
\hline 59.001 & -2.021 & $21.02 \mathrm{Sc}$ III & 197.6 & 2.5 & 169685.9 & 1.5 & $3 d$ & $7 \mathrm{p}$ & No & 59.0010 & & 197.64 & 169685.9 \\
\hline 62.109 & -2.494 & $21.02 \mathrm{Sc}$ III & 25539.3 & 0.5 & 186547.7 & 1.5 & $4 \mathrm{~s}$ & $10 p$ & & & & & \\
\hline 62.114 & -2.827 & $21.02 \mathrm{Sc}$ III & 25539.3 & 0.5 & 186533.9 & 0.5 & $4 \mathrm{~s}$ & $10 \mathrm{p}$ & & & & & \\
\hline 62.707 & -0.591 & $21.02 \mathrm{Sc}$ III & 0.0 & 1.5 & 159472.2 & 2.5 & $3 d$ & $5 f$ & No gf & 62.7070 & -0.550 & 0 . & 159472.24 \\
\hline 62.785 & -1.736 & $21.02 \mathrm{Sc}$ III & 197.6 & 2.5 & 159472.2 & 2.5 & $3 d$ & $5 f$ & $\mathrm{Nr} g f$ & 62.7850 & -1.690 & 197.64 & 159472.24 \\
\hline
\end{tabular}

Table 5. Excerpt for the level list table (see text for explanations).

\begin{tabular}{|c|c|c|c|c|c|c|c|c|c|}
\hline$i$ & $\begin{array}{c}E \\
\left(\mathrm{~cm}^{-1}\right)\end{array}$ & $2 J+1$ & Configuration & $\begin{array}{c}E-E_{\mathrm{FAC}} \\
\left(\mathrm{cm}^{-1}\right)\end{array}$ & $\begin{array}{c}E-E_{\mathrm{NIST}} \\
\left(\mathrm{cm}^{-1}\right)\end{array}$ & $\begin{array}{l}E_{\mathrm{NIST}} \\
\left(\mathrm{cm}^{-1}\right)\end{array}$ & NIST level & & \\
\hline 5 & 218720.00 & 1 & $2 \mathrm{~s} 2.2 \mathrm{p} 2$ & -2653.31 & 0.00 & 218720. & $2 \mathrm{~s} 2.2 \mathrm{p} 2$ & $1 \mathrm{~S}$ & 0 \\
\hline 6 & 301400.00 & -5 & $2 \mathrm{~s} 1.2 \mathrm{p} 3$ & 1037.86 & 0.00 & [301 400] & $2 \mathrm{~s} .(2 \mathrm{~S}) .2 \mathrm{p} 3 .(4 \mathrm{~S} *)$ & $5 \mathrm{~S}^{*}$ & 2 \\
\hline 7 & 536610.00 & -5 & $2 \mathrm{~s} 1.2 \mathrm{p} 3$ & -5843.89 & 0.00 & 536610. & $2 \mathrm{~s} .(2 \mathrm{~S}) .2 \mathrm{p} 3 .(2 \mathrm{D} *)$ & $3 \mathrm{D}^{*}$ & 2 \\
\hline $\begin{array}{ll}\cdots \\
20\end{array}$ & 1451350.00 & $\ddot{1}$ & $2 \mathrm{p} 4$ & -25044.67 & 0.00 & 1451350 . & $2 \mathrm{p} 4$ & $1 \mathrm{~S}$ & 0 \\
\hline 21 & 4593994.30 & -1 & $2 \mathrm{~s} 2.2 \mathrm{p} 1.3 \mathrm{~s} 1$ & -8065.54 & & & & & \\
\hline 22 & 4600160.02 & -3 & $2 \mathrm{~s} 2.2 \mathrm{p} 1.3 \mathrm{~s} 1$ & -8065.54 & -5839.98 & 4606000. & $2 \mathrm{~s} 2.2 \mathrm{p} .3 \mathrm{~s}$ & $3 \mathrm{P} *$ & 1 \\
\hline
\end{tabular}

These comparisons utilized the transition data that we generated with FAC. These data have also been corrected to take into account the adjustments performed on the level energies as explained in Sect. 3.2. Wavelengths, oscillator strengths, and transition probabilities were scaled according to their energy dependences ${ }^{7}$. Owing to the quality of the calculated level energies and our procedure for the energy corrections, modifications to the radiative data are minor.

\section{Description of the data tables}

Our results regarding atomic structure and transition data for the nineteen ions Sc III to Sc XXI are available at CDS in the form of electronic tables. The first table gives a wavelength-ordered list of lines for successive spectra from Sc III to Sc XXI. Only transitions with weighted oscillator strength $g f>10^{-3}$ are listed. Our data refers to the corrected values that we adopted. The total number of transitions is slightly above one million (see eighth column in Table 3). The format is similar to the Kurucz data in order to allow a easy implementation into astrophyical codes. An except of the electronic table is given is Table 4. For each transition: Col. 1 gives the wavelength (in $\mathrm{nm}$ unit, all in vacuum); Col. 2 the weighted oscillator strength; Col. 3 the identification of the spectrum, that is the decimal code (nuclear and ionic charge) and spectroscopic notation; Cols. 3 to 6 the energy ${ }^{8}$ (in $\mathrm{cm}^{-1}$ ) and the total angular momentum $J$ for successively the lower and upper levels; and Cols. 7 and 8, the electronic subshell $n l$ of the outer electron for the lower and upper state of the transition. Additional data in the following columns are given

\footnotetext{
7 The FAC code allows the use of corrected energies (keeping wavefunctions, hence multipolar integrals, fixed) and we checked in a few test cases that the results remained the same.

8 To convert FAC energies to $\mathrm{cm}^{-1}$, we used the value 1. $\mathrm{eV}=8065.54445 \mathrm{~cm}^{-1}$, which corresponds to the "best" value for the ratio of $\mathrm{eV}$ to $\mathrm{cm}^{-1}$ data in NIST.
}

to help the comparison with the experimental data compiled in the NIST database. For transitions between two levels that both correspond to an experimental one, the related NIST data are added: wavelength (Col. 11), $\log (g f)$ if present (Col. 12), and level energies (in $\mathrm{cm}^{-1}$, Cols. 13 and 14). These energies are given with their number of digits as found in NIST. A tag in Col. 10 characterizes the data. The label "No" or "Nr" indicates that the NIST wavelength is either observed or deduced from the Ritz combination. The label "P" indicates a predicted wavelength from two experimental levels, without any corresponding line in NIST, and the label "gf" is added when the $g f$ value is given in NIST.

A second table gives an energy-ordered list of all levels from Sc XXI to Sc III. The correspondence with NIST levels is also given, when it exists, as well as data allowing to go back to our original results. An excerpt is given in Table 5. Column 1 is the level index that will be used in the extended transition file. Column 2 is the adopted level energy (in $\mathrm{cm}^{-1}$ ), and Col. 3 is the degeneracy $2 J+1$ multiplied by -1 for odd levels. The leading configuration of the level is given in Col. 4. Column 5 gives the difference between the adopted value for the energy and the value calculated by FAC. The following columns are present when there is a correspondence to a NIST level: Col. 6 gives the difference between the adopted value and NIST energy $(0.00$ when the level is set to NIST value). Column 7 gives the NIST energy found in the database with its conventions (" $[. .$.$] ": theo-$ retical; " $+x$ ": for systems not connected to the ground state). The NIST level name (configuration, term, and angular momentum) are coded in Col. 8.

Extensive linelists for individual ions are given in separate tables. They provide data for transitions selected with $g A / g A_{\max }>10^{-7}$, where $g A$ is the weighted transition probability and $g A_{\max }$ is the maximum probability for the ion. This amounts to a total of lines of around two million. Transitions are sorted first according to the energy of the upper level, then of 
Table 6. Excerpt of the extended linelists for each ion (see text for explanations).

\begin{tabular}{ccccccccc}
\hline \hline$i_{\mathrm{u}}$ & $(2 J+1)_{\mathrm{u}}$ & $i_{1}$ & $(2 J+1)_{1}$ & $\begin{array}{c}E \\
\left(\mathrm{~cm}^{-1}\right)\end{array}$ & $\log g f$ & Tag & $\begin{array}{c}E_{\mathrm{N}} \\
\left(\mathrm{cm}^{-1}\right)\end{array}$ & $\log g f_{\mathrm{N}}$ \\
\hline 6 & -5 & 2 & 3 & 124143.19 & -3.912 & & & \\
6 & -5 & 3 & 5 & 120907.39 & -3.485 & & & \\
7 & -3 & 1 & 1 & 179962.30 & -1.231 & No gf & 179962.00 & -1.270 \\
7 & -3 & 2 & 3 & 177690.40 & -1.517 & No gf & 177690.40 & -1.550 \\
7 & -3 & 3 & 5 & 174454.60 & -2.973 & Nr gf & 174454.60 & -3.000 \\
7 & -3 & 4 & 5 & 154935.40 & -3.404 & $\mathrm{P}$ & 154935.40 & \\
7 & -3 & 5 & 1 & 125097.90 & -4.247 & $\mathrm{P}$ & 125097.90 & \\
8 & -5 & 2 & 3 & 177760.80 & -0.888 & No gf & 177760.80 & -0.910 \\
\hline
\end{tabular}

the lower level. An excerpt is given in Table 6. Columns 1 to 4 give the index and the degeneracy of the upper level, then of the lower level. The index refers to the entry in the level list table for this ion. Column 5 gives the energy of the transition (in $\mathrm{cm}^{-1}$ ) and Col. 6 its $g f$ value. The tag in Col. 7 gives the same information as in the previous linelist. The wavenumber of the NIST transition is given in Col. 8 (in $\mathrm{cm}^{-1}$ ), its $g f$ value when it exists in Col. 9.

\section{Conclusion}

We computed extensive atomic and radiative data for nineteen ions of scandium using the FAC code. We have identified thousands of levels and millions of lines, which have been made available electronically, including in particular highly excited states that are seldom considered. We took special care to compare our results with the reference data obtained from the NIST databank. Our comparisons show that FAC is able to provide large-scale data of good quality, thus can fill existing gaps in the atomic data at hand for astrophysical purposes. Radiative data have also been compared to the Kurucz linelist for scandium, for which we detected some flaws. We plan in the near future to extend our approach to other poorly studied ions or elements, such as nickel.

Acknowledgements. We acknowledge financial support from the "Programme National de Physique Stellaire" (PNPS) of CNRS/INSU, France, for this work. Part of this work used data obtained from 1995 Atomic Line Data (R. L. Kurucz and B. Bell) Kurucz CD-ROM No. 23. Cambridge, Mass.: Smithsonian Astrophysical Observatory.

\section{Appendix A: Comments about specific ions}

\section{A.1. ScXXI}

The H-like ion has not been observed, and NIST gives theoretical energies and predicted wavelengths without oscillator strengths. For this simple electronic structure, theoretical data are accurate. The FAC code easily reproduces the 25 levels listed by NIST. Besides a shift of $0.063 \mathrm{eV}$ to match the absolute ground level $(6033.755 \mathrm{eV})$, the relative energies are consistent within $0.007 \mathrm{eV}$ (rms of the differences: $0.002 \mathrm{eV}$ ).

\section{A.2. Sc $X X$}

This highly ionized ion has only two observed lines (not in NIST database). As for other He-like structures, this spectra has been the subject of numerous theoretical works. All published energies and transitions are derived from calculations. The NIST data include 25 levels. They show mixing between the ${ }^{1} \mathrm{P}_{1}$ and
${ }^{3} \mathrm{P}_{1}$ systems of about $5 \%$ in the $1 \mathrm{~s} n \mathrm{p}$ configurations. The FAC calculation includes 199 levels, the 25 lowest energies being consistent with the NIST data. The largest discrepancy $(2.2 \mathrm{eV}$ or $0.05 \%$ of the excitation energy) occurs for the $1 \mathrm{~s} 2 \mathrm{p}{ }^{1} \mathrm{P}_{1}^{\circ}$ level, which is affected by the ${ }^{1} \mathrm{P}_{1}-{ }^{3} \mathrm{P}_{1}$ mixing. The $1 \mathrm{~s} 2 l$ level energies were adjusted to those of NIST, the $1 \mathrm{~s} 3 l$ and $1 \mathrm{~s} 4 l$ ones being increased by $0.7 \mathrm{eV}$ and $1.1 \mathrm{eV}$, respectively, and all others by $1.2 \mathrm{eV}$.

\section{A.3. ScXIX and ScXVIII}

For Sc XIx (ground state $1 s^{2} 2 s$ ), we found excellent agreement between the calculated and experimental data. The 27 experimental levels of the Li-like configurations $1 \mathrm{~s}^{2} n l$ are reproduced well by the FAC calculation, the rms of the energy deviation being $0.73 \mathrm{eV}$, which is $0.06 \%$ of the ionization energy. The 8 lower levels were adjusted to NIST values and the $1 \mathrm{~s}^{2} 4 l$ levels raised by $0.3 \mathrm{eV}$. For Sc XVIII (ground state $1 \mathrm{~s}^{2} 2 \mathrm{~s}^{2}$ ), the variance in the deviation from NIST is $0.07 \%$. The 9 lower excited levels belonging to the configurations $1 s^{2} 2 s 2 p$ and $1 s^{2} 2 p^{2}$ were fixed to the experimental energies and the upper levels shifted down by $1 \mathrm{eV}$. The present list of 99 levels for Sc XIX and 676 levels for Sc XvIII help to complete the experimental level list in the low energy part.

For these two simple spectra, the FAC $g f$ values are in good agreement with the compiled ones from NIST. The two Sc XVIII Ritz lines at $\lambda=2.068 \mathrm{~nm}$ and $2.147 \mathrm{~nm}$ are given in NIST as $1 \mathrm{~s}^{2} 2 \mathrm{p}^{2}-1 \mathrm{~s}^{2} 2 \mathrm{~s} 3 \mathrm{p}$ transitions with $\log g f=-1.008$ and 0.21 , respectively. Our calculations give $\log g f=-1.88$ and -3.10 , which corresponds more closely to a two-electron jump.

\section{A.4. Sc XVII to ScXII}

The ground state of theses six ions belongs to configurations $1 \mathrm{~s}^{2} 2 \mathrm{~s}^{2} 2 \mathrm{p}^{n}(n=1$ to 6$)$. The FAC calculation delivers much more levels than present in the NIST lists (see Table 2). It may show significant discrepancy (up to $4 \mathrm{eV}$ ) for the first core-excited configurations of the type $1 \mathrm{~s}^{2} 2 \mathrm{~s} 2 \mathrm{p}^{n+1}$ and $1 \mathrm{~s}^{2} 2 \mathrm{p}^{n+2}$. For Sc XVI, Sc xv, and Sc XIV, the theoretical results of Bromage \& Fawcett (1977c,a,b) were used to confirm the correspondence between the FAC and NIST level identifications, specifically for the levels in the $2 \mathrm{~s}^{2} 2 \mathrm{p}^{n} 3 \mathrm{~d}(n=1,2,3)$ configurations, which are perturbed by the configuration interaction with $2 \mathrm{~s} 2 \mathrm{p}^{n+1} 3 \mathrm{p}$. The Sc xv NIST level $2 \mathrm{~s}^{2} 2 \mathrm{p}^{2}\left({ }^{1} \mathrm{P}\right) 3 \mathrm{~d}^{2} \mathrm{P}_{3 / 2}$ surely does not exist. It is duplicated from the level $2 \mathrm{~s}^{2} 2 \mathrm{p}^{2}\left({ }^{1} \mathrm{D}\right) 3 \mathrm{~d}^{2} \mathrm{P}_{3 / 2}$. We also note that the NIST level of Sc XIII labelled $2 \mathrm{~s}^{2} 2 \mathrm{p}^{4}\left({ }^{3} \mathrm{P}\right) 3 \mathrm{~d}^{4} \mathrm{~F}_{5 / 2}$ (at $492.01 \mathrm{eV}$ ) does not appear in any experimental line.

The levels from the configurations $2 \mathrm{~s}^{2} 2 \mathrm{p}^{n}, 2 \mathrm{~s} 2 \mathrm{p}^{n+1}$ and $2 \mathrm{p}^{n+2}$ were fixed to their experimental values for all these ions, with the 
levels from $2 s^{2} 2 p^{2}\left({ }^{1} S\right) 3 d^{2} D$ for $S c x v$, from $2 s^{2} 2 p^{4} 3 s$ for $S c x I I I$, and from $2 \mathrm{~s}^{2} 2 \mathrm{p}^{5} 3 l$ for Sc XII. Other levels were shifted down by $1.2,1.0,1.0$, and $0.8 \mathrm{eV}$ for Sc xvII, Sc xvI, Sc xv and Sc xIV respectively, and raised by $0.4 \mathrm{eV}$ for Sc XIII.

\section{A.5. ScXI and ScX}

The $n=2$ shell remains closed for these Na-like and Mg-like ions. The Sc XI level $2 \mathrm{p}^{6} 10 \mathrm{p}^{2} \mathrm{P}_{1 / 2}^{\circ}$ is not in the NIST line list. For this same ion, the NIST level $2 \mathrm{p}^{6} 9 \mathrm{f}^{2} \mathrm{~F}_{7 / 2}^{\circ}$ departs from the general behaviour of the f-series. The Sc XI levels up to $5 \mathrm{f}$ are adjusted to NIST, the $5 \mathrm{~g}$ raised by $0.1 \mathrm{eV}$, and upper levels by $0.3 \mathrm{eV}$. For $\mathrm{Sc} \mathrm{x}$, the levels from the configurations $3 \mathrm{~s}^{2}$ to $3 \mathrm{~s} 4 \mathrm{~s}$, $3 \mathrm{p}^{2}$ and $3 \mathrm{p} 3 \mathrm{~d}$ are fixed to NIST; those from $3 \mathrm{~d}^{2}, 3 \mathrm{~s} 4 \mathrm{p}$ to $3 \mathrm{~s} 5 \mathrm{p}$, and $3 \mathrm{p} 4 \mathrm{~s}$ to $3 \mathrm{p} 4 \mathrm{f}$ were shifted down by $0.3 \mathrm{eV}$, and the upper levels raised by $0.2 \mathrm{eV}$.

\section{A.6. ScIX to Sc VI}

The ground state of these four ions belongs to configurations of the type $3 \mathrm{~s}^{2} 3 \mathrm{p}^{n}$ ( $n=1$ to 4 ). Perturbations occur from configuration interactions that mix some of the $3 \mathrm{~s} 3 \mathrm{p}^{n+1}$ and $3 \mathrm{~s}^{2} 3 \mathrm{p}^{n-1} 3 \mathrm{~d}$ states. When dominant compositions are close to $50 \%$, the assigned configuration is indicative only. For instance in Sc vII, the level computed by FAC at $32.612 \mathrm{eV}$ with even parity and $J=1 / 2$ is labelled $3 \mathrm{~s}^{2} 3 \mathrm{p}^{2} 3 \mathrm{~d}$, but it could equally well be the $3 \mathrm{~s} 3 \mathrm{p}^{4}$ configuration (as it appears in NIST).

Kurucz provides line data for these ions. They are based on the level list given in the NIST database but extended to a number of predicted lines that are given with Ritz wavelengths and theoretical $g f$-values. For Sc Ix, Kurucz clearly miscalculates 3 out of the $15 g f$-values found in the NIST data. These three lines all involve the $3 \mathrm{p}^{34} \mathrm{~S}_{3 / 2}$ level of the quadruplet system, which is not connected to the doublet one. In the case of Sc VII, it appears that the calculated $g f$-values given by Kurucz differ significantly from 16 out of the 20 NIST lines and from the FAC ones, the two latter ones being in reasonable agreement. The error in the Kurucz data can be explained from inverted identifications for levels of the perturbed configurations with three electrons in the $n=3$ shell. We note that NIST gives the $3 \mathrm{~s}^{2} 3 \mathrm{p}^{2}\left({ }^{1} \mathrm{D}\right) 3 \mathrm{~d}^{2} F_{5 / 2}$ level of Sc viI (at $46.876 \mathrm{eV}$ ) without any corresponding line.

The list of experimental levels for Sc vi is incomplete for many configurations and there is no $g f$-value in NIST. For the $3 \mathrm{~s}^{2} 3 \mathrm{p}^{3} 3 \mathrm{~d}$, configuration NIST gives 12 levels among the $38 \mathrm{ex}-$ pected ones calculated by FAC. The only observed transitions are from the ground state. They allow us to determine levels up to $J=3$ only, hence the high- $J$ levels are missing. From the FAC results, we can predict numerous strong transitions $3 s^{2} 3 p^{3} 3 d-3 s^{2} 3 p^{3} 4 f$ between high- $J$ levels $(J=4$ to 6$)$ in the wavelength range $20-30 \mathrm{~nm}$. Other strong predicted transitions in $3 s^{2} 3 p^{3} 3 d-3 s^{2} 3 p^{2} 3 d^{2}$ appear in the same range, owing to the interaction between the overlapping configurations $3 \mathrm{~s}^{2} 3 \mathrm{p}^{3} 4 \mathrm{f}$ and $3 \mathrm{~s}^{2} 3 \mathrm{p}^{2} 3 \mathrm{~d}^{2}$. NIST lists 4 unclassified lines of Sc vi that could be due to $3 s^{2} 3 p^{3} 3 d-3 s^{2} 3 p^{3} 4 f$. Such a tentative classification is reported by the Kelly compilation (Kelly \& Palumbo 1973), but the corresponding levels values are unreliable because of the lack of connection to known levels of $J$-values lower than 3 .

For Sc IX, the energies of the twelve levels from $3 \mathrm{~s}^{2} 3 \mathrm{p}, 3 \mathrm{~s} 3 \mathrm{p}^{2}$ and $3 s^{2} 3 d$ were adjusted to the values of NIST, and the upper levels shifted down by $0.3 \mathrm{eV}$ (see Fig. 1). For Sc viII, levels from $3 \mathrm{~s}^{2} 3 \mathrm{p}^{2}, 3 \mathrm{~s} 3 \mathrm{p}^{3}$ and $3 \mathrm{~s}^{2} 3 \mathrm{p} 3 \mathrm{~d}$ were adjusted to NIST, except for the missing levels $3 \mathrm{~s} 3 \mathrm{p}^{3}{ }^{5} \mathrm{~S}_{2}$ (shifted down by $0.6 \mathrm{eV}$ ) and $3 \mathrm{~s}^{2} 3 \mathrm{p} 3 \mathrm{~d}^{1} \mathrm{D}_{2},{ }^{3} \mathrm{~F}_{1,2,3}$ (shifted down by $1.4 \mathrm{eV}$ ). For $\mathrm{Sc}$ vII, all levels with a correspondence to NIST were adjusted to their NIST value, the remaining levels from $3 \mathrm{~s}^{2} 3 \mathrm{p}^{2} 3 \mathrm{~d}$ shifted down by $2.0 \mathrm{eV}$, and other levels by $0.5 \mathrm{eV}$. For Sc VI, all levels from $3 s^{2} 3 p^{4}, 3 s 3 p^{5}, 3 s^{2} 3 p^{3} 3 d$ and $3 s^{2} 3 p^{3} 4 s$, with a correspondence to NIST were adjusted to the energies given by NIST, and the remaining levels from $3 \mathrm{~s}^{2} 3 \mathrm{p}^{3} 3 \mathrm{~d}$ were shifted down by $1.0 \mathrm{eV}$, and other levels by $2.5 \mathrm{eV}$.

\section{A.7. Sc V}

The NIST database gives 118 Sc v energy levels and 454 electric dipolar transitions, derived from the analysis of Smitt \& Ekberg (1985). Theoretical oscillator strengths for K III, Ca IV, and Sc V iosoelectronic spectra were published by Wilson et al. (2000). We note that the Kurucz data for Sc v should be updated: the 36 listed lines are based on only 22 levels. Kurucz denominations for the parent terms $3 p^{4}\left({ }^{3} \mathrm{P}\right)$ and $3 \mathrm{p}^{4}\left({ }^{1} \mathrm{D}\right)$ in the $3 \mathrm{p}^{4} 3 \mathrm{~d}$ configuration should be inverted. The four levels from $3 \mathrm{p}^{4} 5 \mathrm{~s}$ do not match any NIST level.

We established a complete level correspondence between FAC and NIST for the two overlapping odd configurations $3 s^{2} 3 p^{4} 4 p$ and $3 s 3 p^{5} 3 d$, which have 21 and 23 levels, respectively. Smitt \& Ekberg (1985) highlighted their mixed composition, with additional perturbation by the numerous unobserved levels of the $3 \mathrm{~s}^{2} 3 \mathrm{p}^{3} 3 \mathrm{~d}^{2}$ configuration. The NIST level $3 \mathrm{~s} 3 \mathrm{p}^{5}\left({ }^{3} \mathrm{P}\right) 3 \mathrm{~d}^{4} \mathrm{D}_{5 / 2}$ is labelled $4 \mathrm{p}$ by FAC, the mixing being calculated as $48 \%-34 \%$ by Smitt \& Ekberg (1985). On the even side, the 72 levels from the configurations $3 s^{3} 3 p^{6}, 3 s^{2} 3 p^{4} 3 d, 3 s^{2} 3 p^{4} 4 s$, $3 \mathrm{~s}^{2} 3 \mathrm{p}^{4} 4 \mathrm{~d}$, and $3 \mathrm{~s}^{2} 3 \mathrm{p}^{4} 5 \mathrm{~s}$ are observed and matched to the FAC levels, except for the $3 s^{2} 3 p^{4}\left({ }^{3} \mathrm{P}\right) 5 \mathrm{~s}^{4} \mathrm{P}_{5 / 2}$ level, which is unobserved. The energies of all the levels with a correspondence were adjusted to the values of NIST, and other levels shifted down by $2.0 \mathrm{eV}$, except for the level $3 \mathrm{~s}^{2} 3 \mathrm{p}^{4} 5 \mathrm{~s}^{4} \mathrm{P}_{5 / 2}$ which was shifted down by $1.6 \mathrm{eV}$.

\section{A.8. ScIV}

All the 127 NIST levels were related to the FAC levels (the NIST level $3 \mathrm{~s} 3 \mathrm{p}^{6} 5 \mathrm{p}^{1} \mathrm{P}^{*}$, above the ionization limit, is not considered). The theoretical excitation energies from the closed ground state configuration $3 s^{2} 3 p^{6}$ are systematically higher than experimental ones by about $2 \mathrm{eV}$. An exception is the highest level ${ }^{1} \mathrm{P}_{1}$ of the $3 p^{5} 3 d$ configuration, which deviates from the observed one by $4 \mathrm{eV}$. This level is separated from the rest of its configuration by the 4 levels of $3 p^{5} 4 s$. The unobserved configuration $3 p^{4} 3 d^{2}$ is predicted to overlap with the $3 p^{5} 5 p$ and $3 p^{5} 4 f$ ones. The NIST level $3 \mathrm{p}^{5} 4 \mathrm{~d}^{1} \mathrm{P}^{*}$ is given by FAC as an extra $3 \mathrm{p}^{5} 5 \mathrm{~s}$ level, the label being meaningless in the case of large mixing. Kurucz $1204 \mathrm{gf}$ values differ substantially from our results. No clear conclusion can be drawn as only 4 NIST transitions are available. The energies of the levels from $3 p^{6}$ to $3 p^{5} 5 p$ and $3 s 3 p^{6} 3 d$ are adjusted to NIST, and those of other levels were shifted down by $1.45 \mathrm{eV}$.

\section{A.9. Sc III}

The structure of this simple ion is based on the closed core $3 s^{2} 3 p^{6}$. The 97 NIST $g f$ values are nicely reproduced by FAC. In contrast the $g f$ values given by FAC and Kurucz differ for many of the supplementary 129 lines provided by Kurucz. All NIST energies were used to adjust those FAC levels where a correspondence was found and other levels were raised by $1.2 \mathrm{eV}$. 
G. Massacrier and M.-C. Artru: Extensive spectroscopic data for ionized scandium

\section{References}

Aggarwal, K. M., Keenan, F. P., \& Heeter, R. F. 2010, Phys. Scripta, 81, 015303 Aggarwal, K. M., Kato, T., Keenan, F. P., \& Murakami, I. 2011, Phys. Scripta, 83, 015302

Bromage, G. E., \& Fawcett, B. C. 1977a, MNRAS, 178, 605

Bromage, G. E., \& Fawcett, B. C. 1977b, MNRAS, 179, 683

Bromage, G. E., \& Fawcett, B. C. 1977c, MNRAS, 178, 591

Clementson, J., Beiersdorfer, P., \& Gu, M. F. 2010, Phys. Rev. A, 81, 012505

Gu, M. F. 2003, ApJ, 582, 1241

Gu, M. 2005, Atomic Data and Nuclear Data Tables, 89, 267

Gu, M. F. 2008, Canadian J. Phys., 86, 675

Hahn, M., Bernhardt, D., Lestinsky, M., et al. 2010, ApJ, 712, 1166

Hibbert, A., Johnson, W., \& Wiese, W. 2010, J. Phys. B: Atom. Mol. Opt. Phys., 43, 070201

Kaufman, V., \& Sugar, J. 1988, J. Phys. Chem. Ref. Data, 17, 1679
Kelly, R., \& Palumbo, L. 1973, Naval Research Laboratory Report, No 7599 LeBlanc, F., \& Alecian, G. 2008, A\&A, 477, 243

Ralchenko, Y., Kramida, A., Reader, J., \& NIST ASD Team. 2010, NIST Atomic Spectra Database (version 4.0), Tech. rep., National Institute of Standards and Technology, Gaithersburg, MD.

Rodrigues, G. C., Indelicato, P., Santos, J. P., Patté, P., \& Parente, F. 2004 Atomic Data and Nuclear Data Tables, 86, 117

Rogers, F. J., \& Iglesias, C. A. 1992, ApJ, 401, 361

Seaton, M. J., Zeippen, C. J., Tully, J. A., et al. 1992, Rev. Mex. Astron. Astrofis., 23, 19

Seaton, M. J., Yan, Y., Mihalas, D., \& Pradhan, A. K. 1994, MNRAS, 266, 805 Smitt, R., \& Ekberg, J. O. 1985, Phys. Scr, 31, 391

Sossah, A. M., Zhou, H., \& Manson, S. T. 2008, Phys. Rev. A, 78, 053405

Sugar, J., \& Corliss, C. 1980, J. Phys. Chem. Ref. Data, 9, 473

Wei, H. G., Shi, J. R., Zhao, G., \& Liang, Z. T. 2010, A\&A, 522, A103

Wilson, N. J., Hibbert, A., \& Bell, K. L. 2000, Phys. Scripta, 61, 603 